\title{
Takotsubo syndrome triggered by emotional stress: a case report
}

\section{Emine Mülayim •}

Department of Psychiatry, Kahramanmaraş Necip Fazıl City Hospital, Kahramanmaraş, Turkey

\begin{abstract}
Takotsubo syndrome is an acute cardiac syndrome characterized by transient systolic dysfunction of the left ventricle without coronary artery disease. It's etiology has not been fully explained, physical and intense emotional stress triggers this syndrome so it is also called "broken heart syndrome" or "stress cardiomyopathy". Takotsubu syndrome has been reported that approximately $2 \%$ of patients presenting with acute coronary syndrome. In this case, we presented a 65 -year-old patient who had a Takotsubo syndrome attack after emotional stress, thinking that it would contribute to the literature and increase awareness of this disease.

Keywords: Takotsubo syndrome, stress cardiomyopathy, catecholamine, emotional stress, somatic stress
\end{abstract}

$\mathrm{T}$ akotsubo syndrome (TS) is an acute cardiac syndrome characterized by transient left ventricular systolic dysfunction without coronary artery disease [1]. It was first reported in Japan by Sato et al. [2] in the 1990's. Although its etiology is not fully explained, it is known that sudden and intense emotional stress triggers this syndrome [3]. This is why it is also called "broken heart syndrome" or "stress cardiomyopathy". There are somatic and emotional stress factors defined for this disease in Table 1 [4]. It is frequently observed in women and in the post-menopausal period [5].

It has been reported that approximately $2 \%$ of patients presenting with acute coronary syndrome have TS [5]. The psychiatric aspect of TS, which is encountered in cardiology and anesthesia clinics and whose treatment is regulated by these clinics, is bypassed. Here, I present a rare TS case that develops due to stress to draw attention to the psychiatric aspect of the disease.

\section{CASE PRESENTATION}

Our patient was a 65 years old female patient, a retired teacher. The patient came for a psychiatric examination at the request of her children, who were physicians. She had complaints of concern, anxiety, irritability, and sleep disturbance. Her children stated that their mother was very prissy and could not tolerate a mess at home. However the main reasons for bringing the patient to the examination, it was after the patient learned that she was diagnosed with takotsubo syndrome during a detailed cardiological examination.

When the anamnesis was deepened, it was learned that the psychiatric complaints of the patient started in her youth. She lost her father at an early age. Afterwards, she started to get angry, stress, distress, sleep disturbance, occasional palpitations, and shortness of breath. She occasionally had crying jags. She had no lack of enjoyment and unhappiness in life. She was 


\begin{tabular}{ll}
$\begin{array}{l}\text { Table 1. Preceding somatic or emotional } \\
\text { cardiomyopathy [4] }\end{array}$ & Emotional stressors \\
\hline Somatic stressors & Grief (eg, death or illness of a loved one) \\
\hline Vigorous exercise & $\begin{array}{l}\text { Receiving bad news (eg, being diagnosed with } \\
\text { major illness, learning of a daughter's divorce) }\end{array}$ \\
\hline $\begin{array}{l}\text { Pheochromocytoma } \\
\text { Subarachnoid hemorrhage }\end{array}$ & Fear \\
\hline Seizure & Relationship conflicts \\
\hline Postoperative pain & Public speaking \\
\hline Hyperthyroidism & Financial problems \\
\hline Alcohol/opiate withdrawal & Being bullied \\
\hline Invasive medical procedures & Surprise party \\
\hline Exacerbation of underlying noncardiac disease & Changing residence involvement in accident \\
\hline Sexual intercourse & \\
\hline Administration of sympathomimetics & \\
\hline
\end{tabular}

also a prissy person at that time, doing thorough cleaning once a week. She did not get help because she thought that no one else could clean as she did. On the other days, she was very careful about her cleanliness and order and could not tolerate mess. She was always a stubborn person, and no one else could change what she knew right.

The patient had a heart attack. In the evening of her routine weekly cleaning, her neighbor and little child came to visit without notice. The little boy messed up the cleaned house and soiled it. The patient was very angry with this situation, and distress started first. Chest tightness, palpitations, and shortness of breath were started then. Her complaints, which she had ignored before, increased over time. Chest pain intensified, palpitations, shortness of breath increased, left arm numbness, cold sweating occurred.

They were admitted to the emergency department and, after the first laboratory examinations and ECG, she was told that she had a heart attack, and was taken to the intensive care unit. Coronary artery occlusion was not detected in the angiography performed. Echocardiography revealed left ventricular apical dyskinesia (aneurysmatic), ejection fraction $60 \%$, and mild mitral insufficiency. The patient was treated in the intensive care unit for three days. And then, cardiac treatment was arranged, saying that heart failure de- veloped secondary to the attack. Control angiography was recommended for subsequent follow-ups. She was examined by a different cardiologist after her discharge. Coronary artery occlusion was not observed in the patient who underwent angiography again, and no other disease was detected in the patient at that time. Considering the prior angiography and laboratory findings, it was stated that the acute coronary attack she experienced in the past was TS, a condition that may develop due to stress.

In the mental state examination, her general appearance was compatible with her age, and her selfcare was complete. Her attitude during the interview was communicative, and she made eye contact. Her affect was tense, and her mood was anxious. Her concentration of attention, orientation, judgment, and abstract thinking was complete. No perceptual disorder was detected.

The patient was diagnosed with a generalized anxiety disorder and obsessive-compulsive personality disorder according to DSM 5 diagnostic criteria [6]. And sertraline $50 \mathrm{mg}$ and mirtazapine $15 \mathrm{mg}$ were started. Since her complaints did not regress during her follow-up, sertraline dosage was increased to 100 mg. The patient, who stated that she started to gain weight over time, and her sleep improved, mirtazapine was discontinued, and her treatment with sertraline 
$100 \mathrm{mg}$ was continued. She was followed up in the psychiatry outpatient clinic for a year. And the patient's cardiac attack did not recur.

\section{DISCUSSION}

TS is diagnosed according to Mayo clinic diagnostic criteria. ECG findings of patients, who present with complaints of chest pain, shortness of breath, palpitations similar to acute myocardial infarction, reveal T negativity and ST elevation, and a moderate elevation in troponin levels occur [7]. Differential diagnosis from acute myocardial infarction can be made by angiography [8]. Although there is no stenosis in the coronary artery, there is temporary dyskinesia or akinesia in the apical and middle segments of the left ventricle. This condition creates the appearance of a narrow neck and apical ballooning at the end of the systole in the left ventricle. The name Takotsubo was given because the appearance of the heart in echocardiography was compared to the shape of the container the Japanese used to catch octopus [7]. Although TS is thought to have a relatively benign prognosis, ventricular fibrillation can be observed in cardiogenic shock [7].

The typical feature of TS is postmenopausal woman and emotional or somatic stress that precede the onset of cardiothoracic symptoms [4, 9]. Studies have reported the prevalence of anxiety and depression as $56 \%$ and $48 \%$ in these patients [10]. Similarly, it was observed in our patient that she had a generalized anxiety disorder and obsessive-compulsive personality traits before TS appeared. Just before the postmenopasual female patient had an acute cardiac attack, she experienced an event that caused an increase in stress. During her emergency admission, she was followed up and treated considering acute myocardial infarction. Coronary artery disease was not detected in the angiography performed for the patient. Echocardiography showed apical ballooning in the left ventricle, which is diagnostic for TS. In this respect, our patient had typical TS.

The patient has a psychiatric disorder that has existed for a long time. However, she did not accept this situation and did not receive any psychiatric support. Not sharing negative feelings after stressful events in these patients may cause exaggerated sympathetic and cardiovascular reactions [11]. And, exposure to an internal or external stimulus that overwhelms their psychological coping ways in these patients can cause somatic complaints. In other words, they can respond to a psychological threat as a physiological threat [11]. The stressful event experienced in our patient also caused an excessive sympathetic reaction physiologically.

The pathophysiology of TS is complex. It includes cardiovascular responses to endogenous catecholamines or to exogenously administered catecholamines, often spiked during acute severe stress. Therefore, catecholamine concentration is shown as a trigger factor in TS [12]. Characteristic morphological changes similar to cardiotoxic effects caused by catecholamines were also observed in cardiac biopsies taken in the acute phase of the patients [13].

The stress response in the human body is managed through both the central nervous system and the sympathetic nervous system. Somatic or emotional stress causes catecholamine release in the central nervous system. The basic anatomical structures that play a role in stress response are the limbic system, neocortex, spinal cord, reticular formation, and brainstem. Locus coeruleus serves as the central area of neurons in the sympathetic nervous system. And it can receive afferent signals from the amygdala, hypothalamus, and cingulate gyrus. It is about excitement and alertness during stress [12].

Neuroendocrine changes caused by stimulation of these structures include intense stimulation of the locus coeruleus-adrenomedullin axis and hypothalamus-pituitary-adrenocortical axis. Postganglionic fibers of the sympathetic nervous system extend along the epicardial vessels, affecting the myocardial and coronary circulation [12]. As a result, two stages are mentioned in TS cases triggered by emotional stress. The first stage begins with the increased release of epinephrine and norepinephrine initiated by the cognitive centers of the brain through the activation of the hypothalamic-pituitary-adrenal axis. In the second phase, a cardiovascular response occurs to increased catecholamines in the circulation [5].

Anxiety and chronic stress have been found to increase the likelihood of developing TS significantly [14, 15]. However, this situation is considered to serve not as 
an isolated cause of TS, but to increase susceptibility to the disease. It is because resting catecholamine levels in these individuals fluctuate higher in response to stressors [5].

TS cases accompanying dissociative amnesia and acute manic episode of bipolar disorder have also been reported in the literature $[16,17]$. In addition, TS cases due to the electroconvulsive therapy and psychotropic drugs, which are frequently used in the treatment of patients in psychiatry clinics, have also been reported $[18,19]$. Although the event that triggers TS is often attributed to the psychiatric condition, no detailed information has been obtained about how the clinical approach to these patients will be.

\section{CONCLUSION}

This case shows that TS may be a syndrome closely related to psychiatric diseases. Although it seems necessary that the primary treatment should be regulated by cardiology and anesthesia clinics due to the presence of an acute coronary attack, it shows that the patient may need psychiatric support afterwards.

\section{Authors' Contribution}

Study Conception: EM; Study Design: EM; Supervision: EM; Funding: EM; Materials: EM; Data Collection and/or Processing: EM; Statistical Analysis and/or Data Interpretation: EM; Literature Review: EM; Manuscript Preparation: EM and Critical Review: EM.

\section{Informed consent}

Written informed consent was obtained from the patient for publication of this case and any accompanying images.

\section{Conflict of interest}

The authors disclosed no conflict of interest during the preparation or publication of this manuscript.

\section{REFERENCES}

1. Prasad A, Lerman A, Rihal CS. Apical ballooning syndrome (Takotsubo or stress cardiomyopathy): a mimic of acute myocardial infarction. Am Heart J 2008;155:408-17.
2. Sato H, Tateishi H, Uchida T, Kodama K, Hon M. Takotsubo type cardiomyopathy due to multivessel spasm. In: Kodama K, Haze K, Hon M, editors. Clinical aspect of myocardial injury: from ischemia to heart failure. Kagaku Hyoronsha; Tokyo;1990: pp. 56-64. [in Japanese]

3. Nayeri A, Rafla-Yuan E, Farber-Eger E, Blair M, Ziaeian B, Cadeiras M, et al. Pre-existing psychiatric illness is associated with increased risk of recurrent Takotsubo cardiomyopathy. Psychosomatics 2017;58:527-32.

4. Redfors B, Shao Y, Omerovic E. Stress-induced cardiomyopathy (Takotsubo) broken heart and mind? Vasc Health Risk Manag 2013;9:149-54.

5. Akashi YJ, Nef HM, Lyon AR. Epidemiology and patho-physiology of Takotsubo syndrome. Nat Rev Cardiol 2015;12:38797.

6. American Psychiatric Association. Diagnostic and statistical manual of mental disorders (5th ed.). Arlington, VA: American Psychiatric Press. 2013.

7. Oliveri F, Goud HK, Mohammed L, Mehkari Z, Javed M, Althwanay A, et al. Role of depression and anxiety disorders in Takotsubo Syndrome: the psychiatric side of broken heart. Cureus 2020:12:e10400.

8. Y-Hassan S, Tornvall P. Epidemiology, pathogenesis and management of Takotsubo syndrome. Clin Auton Res 2018;28:53-65. 9. Elsayed M, Connemann BJ, Dahme T, Tesfay T, Gahr M. Takotsubo cardiomyopathy with inconspicuous initial electrocardiogram: a potentially serious cardiac pathology related to emotional stress. Front Pschiatry 2019;10:308.

10. Summers MR, Lennon RJ, Prasad A. Pre-morbid psychiatric and cardiovascular diseases in apical ballooning syndrome (Takotsubo/ stress-induced cardiomyopathy): potential pre-disposing factors? J Am Coll Cardiol 2010;55:700-1.

11. Jenab Y, Hashemi SR, Ghaffari-Marandi N, Zafarghandi H, Shahmansouri N. Psychological features of Takotsubo cardiomyopathy: report of four cases. J Tehran Heart Cent 2017;12:95-8. 12. Wang X, Pei J, Hu X. The brain-heart connection in Takotsubo syndrome: the central nervous system, sympathetic nervous system, and catecholamine overload. Cardiol Res Pract 2020;2020:4150291.

13. Nef HM, Möllmann H, Kostin S, Troidl C, Voss S, Weber M, et al. Takotsubo cardiomyopathy: intraindividual structural analysis in the acute phase and after functional recovery. Eur Heart $\mathrm{J}$ 2007;28:2456-64.

14. Deshmukh A, Kumar G, Pant S, Rihal C, Murugiah K, Mehta JL. Prevalence of Takotsubo cardiomyopathy in the United States. Am Heart J 2012;164:66-71.

15. El-Sayed AM, Brinjikji W, Salka S. Demographic and comorbid predictors of stress (Takotsubo) cardiomyopathy. Am J Cardiol 2012;110:1368-72.

16. Toussi A, Bryk J, Alam A. Forgetting heart break: a fascinating case of transient left ventricular apical ballooning syndrome associated with dissociative amnesia. Gen Hosp Psychiatry 2014;36:225-7.

17. Maldonado JR, Pajouhi P, Witteles R. Broken heart syndrome (Takotsubo cardiomyopathy) triggered by acute mania: a review and case report. Psychosomatics 2013;54:74-9.

18. Chandra PA, Golduber G, Chuprun D, Chandra AB. Takot- 
subo cardiomyopathy following electroconvulsive therapy. J Cardiovas Med 2009;10:333-5.

19. Verduin MI. Commentary on 2 cases of Takotsubo cardiomy- opathy involving psychotropic medication. J Psychiatr Pract 2016;22:239-40. 